\title{
Effect of Single Dose of Radioiodine Therapy on Volume Reduction of Thyroid Gland in $H_{2}$ yperthyroidism

\author{
Department of Nuclear Medicine, United Hospital Limited, Dhaka. \\ ${ }^{2}$ Vice-Chancellor, Bangladesh University of Health Sciences, Darus Salam, Mirpur, Dhaka \\ 3 National Institute of Nuclear Medicine \& Allied Sciences (NINMAS), BAEC, Bangabandhu Sheikh Mujib Medical University campus, Shahbag, Dhaka, 1000.
} \\ ${ }^{1}$, Faridul Alam ${ }^{2}$, Fatima Begum ${ }^{3}$, Sadia Sultana ${ }^{3}$, Zeenat Jabin ${ }^{3}$ and Nurun Nahar ${ }^{3}$
}

Correspondence Address: Shamrukh Khan, Department of Nuclear Medicine, United Hospital Limited, Dhaka, shamrukh@yahoo.com

\begin{abstract}
Introduction: Radioactive iodine therapy (RAIT) in patients with hyperthyroidism (HT) causes apoptosis of thyrocytes to bring about restoration of thyroid function. The aim of the study was to find the short term extent of reduction of thyroid gland volume (TGV) by non-invasive quantitative assessment using ultrasound imaging (USG).

Patients and Methods: This prospective study was conducted on a group of patients who had received RAIT due to primary hyperthyroidism at National Institute of Nuclear Medicine \& Allied Sciences (NINMAS). Pre-therapy work up included hormone assay and baseline measurement of TGV by US before administration of appropriate fixed dose RAIT. Short term follow-up with hormone assay and serial measurements of TGV on two occasions were done at three and six months following the RAIT. Observed temporal changes of parameters were analyzed using appropriate statistics.

Results: Total 117 patients with primary hyperthyroidism had received RAIT with diagnosis of diffuse toxic goiter in 86 patients, toxic multinodular goiter in 21 cases and single toxic nodular goiter in $\mathbf{1 0}$ cases. There was a decline of mean TGV from the baseline level of $24 \mathrm{ml}$ to $14 \mathrm{ml}$ at three months followed by a further decline to 9.1 $\mathrm{ml}$ at six months. Thus the volume reduction of thyroid gland was calculated to be $42 \%$ at three months and $62 \%$ at six months. The volume reduction was observed to be in a correlative trend with the normalization of hormone levels. The proportion of patients who showed persistent hyperthyroidism till the study end point was $23 \%$. Conclusion: Single dose of radioactive iodine therapy resulted in reduction of TGV up to $62 \%$ till six months after RAIT while $23 \%$ patients showed persistent hyperthyroidism. The correlative trend of volume reduction with normalization of hormone levels indicates potentiality of TGV to emerge as an adjunct to conventional assessment of treatment efficacy following RAIT.
\end{abstract}

Key words: Radioiodine therapy, Thyroid gland volume, Hyperthyroidism, High resolution ultrasound.

Bangladesh J. Nucl. Med. Vol. 21 No. 2 July 2018 Doi : https://doi.org/10.3329/bjnm.v21i2.40357

\section{INTRODUCTION}

Radioactive iodine therapy (RAIT) for management of hyperthyroidism (HT) has been practiced in Bangladesh for four decades (1) with reports of satisfactory short-term outcome $(2,3)$. Incidence of HT was found to be $0.86 \%$ in a community based cross sectional study on 925 individuals (4). The common clinical manifestations include diffuse toxic goiter or Graves' disease (GD), single toxic nodular goiter (STG) and toxic multinodular goiter (TMNG) (5). Management with RAIT in all cases of HT is feasible irrespective of age including the pre-pubertal (6) and is considered safe since studies on large number of patients administered with RAIT, followed up for considerably long durations have never demonstrated increased risk of mutagenesis or carcinoma (7, 8). RAIT has long been a recommended management strategy for HT with a higher preference over anti-thyroid drug (ATD) and surgery (9). RAIT lacks recurrence as in case with ATD (10) as well as it lacks morbidities and poor cosmetic outcome unlike surgical intervention (11).

RAIT is selectively taken by sodium iodine symporter of thyroid epithelial cell and causes cell death by beta emission that ensue reduction in size of thyroid gland $(12,13)$. Nygaard et al. observed a decrease of thyroid gland size after RAIT in both toxic and nontoxic goiters (14). Assessment of change of thyroid gland size by non-invasive measurement of thyroid gland volume (TGV) is thus believed to be an important 
adjunct to clinical assessment of efficacy after treatment with RAIT.

HRUS is validated as gold standard for assessment of TGV $(15,16)$ and a recommended diagnostic method for assessment of goiter by World Health Organization (WHO) and International Council for the Control of Iodine Deficiency Disorders (ICCIDD) because high resolution ultrasound (HRUS) facilitates precise delineation of gland with adequate characterization of glandular parenchyma (17).

Thus, this study aimed to observe volume change of thyroid gland after radioiodine therapy by HRUS as an advantageous non-invasive modality.

\section{PATIENTS AND METHODS}

This prospective study was conducted on a group of patients that included all primary hyperthyroid patients who had undergone RAIT after being referred to thyroid division of National Institute of Nuclear Medicine \& Allied Sciences (NINMAS), BAEC. Measurement of TGV with high resolution of ultrasound imaging (HRUS) was done in each patient on three occasions. The baseline measurements were taken before administering RAIT when each patient also had to undergo a pre-defined structured clinical assessment that including symptoms, physical examination and recording of vital signs, assessment of blood levels of free thyroxine (FT4), thyrotrophine (TSH), planar thyroid scan with $99 \mathrm{mTc}$ pertechnetate and radioactive iodine uptake (RAIU). All patients then underwent RAIT with modified fixed dose protocol according to institutional standard of practice. Post RAIT assessment of TGV by HRUS then done on two occasions while all the patients were undergoing a routine protocol-wise three monthly follow up till six months after RAIT. Assay for FT4, TSH were done during each follow-up. Reduction of TGV was calculated by subtracting the post RAIT measurement from that of the pre RAIT measurement in each patient. HRUS of neck with a $7.5 \mathrm{MHz}$ linear transducer with appropriate gain setting was used in real time equipment (Toshiba Core Vision Pro, Tokyo,
Japan). During imaging patient was positioned supine with hyper-extended neck by a pillow under the shoulders. The thyroid gland was identified and visual assessment was done for outline delineation and parenchymal echo-characterization. Each lobe was scanned in both transverse and longitudinal plane in order to estimate its length, width and thickness. Volume of each lobe was calculated done by ellipsoid method using appropriate formula. The volumes of both lobes were then added to calculate the total TGV. Isthmus and pyramidal lobe (if present) were ignored.

Continuous data was expressed as mean and standard deviation (SD), median and value ranges. Categorical data was presented as frequency and percentage. Analysis of variance (ANOVA) of with post-hoq comparison through Hochberg test was done for comparison of $\mathrm{TGV}$ reduction among various clinical groups of hyperthyroidism. Two-sided $p$ value $<0.05$ was considered as statistically significant.

\section{RESULTS}

Total 117 patients with mean age of $42.7 \pm 12.6$ years (median 42 years and range 15 - 77 years) had undergone RAIT. Among them 42 were male and 75 were female. Table 1 shows distribution of age and sex among the study population. The clinical diagnoses among patients undergoing RAIT were diffuse toxic goiter (GD) in $86(73.5 \%)$, single toxic nodular goiter (STG) $10(8.5 \%)$ and toxic multiple nodular goiter (MNG) in 21(17.9\%).

Table 1: Distribution of age and sex of the study population $(n=117)$

\begin{tabular}{|l|l|l|}
\hline Age & Frequency & Percent \\
\hline Below 30 years & 22 & 18.8 \\
\hline $30-39$ years & 21 & 17.9 \\
\hline $40-49$ years & 37 & 31.7 \\
\hline $50-59$ years & 22 & 18.8 \\
\hline 60 years or above & 15 & 12.8 \\
\hline Total & 117 & 100 \\
\hline Sex & & \\
\hline Male & 42 & 35.9 \\
\hline Female & 75 & 64.1 \\
\hline Total & 117 & 100 \\
\hline
\end{tabular}


All patients after receiving RAIT in standard dose were under regular institutional protocol of follow up with serial hormone assay after three and six months. At first follow-up 41\% (48) were euthyroid, 28.2\%

(33) were hypothyroid and $30.8 \%$ (36) were found hyperthyroid. At 2nd follow-up 53.8\% (63) were found euthyroid, $23.1 \%$ (27) were hypothyroid and another $23.1 \%$ (27) were found hyperthyroid. RAIT response among three categories of hyperthyroidism is provided in Table 2 .

Table 2: Comparison of thyroid status in three types of hyperthyroidism.

\begin{tabular}{|c|c|c|c|c|}
\hline & \multicolumn{2}{|l|}{ At 3 months } & \multicolumn{2}{|l|}{ At 6 months } \\
\hline & Frequency & Percent & Frequency & Percent \\
\hline \multicolumn{5}{|c|}{ Diffuse Toxic Goiter } \\
\hline Euthyroid & 38 & 44.2 & 45 & 52.3 \\
\hline Hypothyroid & 28 & 32.6 & 17 & 19.8 \\
\hline Hyperthyroid & 20 & 23.3 & 24 & 27.9 \\
\hline Total & 86 & 100.0 & 86 & 100.0 \\
\hline \multicolumn{5}{|c|}{ Toxic Multinodular goiter } \\
\hline Euthyroid & 8 & 38.1 & 12 & 57.1 \\
\hline Hypothyroid & 4 & 19.0 & 7 & 33.3 \\
\hline Hyperthyroid & 9 & 42.9 & 2 & 9.5 \\
\hline Total & 21 & 100.0 & 21 & 100.0 \\
\hline \multicolumn{5}{|c|}{ Single Toxic Nodular Goiter } \\
\hline Euthyroid & 2 & 20.0 & 6 & 60.0 \\
\hline Hypothyroid & 1 & 10.0 & 3 & 30.0 \\
\hline Hyperthyroid & 7 & 70.0 & 1 & 10.0 \\
\hline Total & 10 & 100.0 & 10 & 100.0 \\
\hline
\end{tabular}

The cumulative data revealed that, during the 1 st follow up there was an increase of mean TSH level with a concomitant reduction of mean TGV in comparison to the baseline. During the 2nd follow up there was however a decrease of mean TSH level with a further concomitant reduction of TGV in comparison to the 1st follow up. For the entire group of study patients the mean TSH at baseline was $0.2 \pm 0.34 \mathrm{~m} \mathrm{IU} / \mathrm{L}$, increasing to mean of $13.6 \pm 0.26 \mathrm{~m} \mathrm{IU} /$ Lat 1 st followup and then a decline to $7.1 \pm 1.7 \mathrm{~m} \mathrm{IU/L}$ at 2 nd followup. The laboratory normal range for TSH assay was 0.3 to $5 \mathrm{~m} \mathrm{IU} / \mathrm{L}$. For the entire group of study patients the mean TGV at baseline was $24.0 \pm 22.9 \mathrm{ml}$, reduced to $14.0 \pm 12.5 \mathrm{ml}$ at $1 \mathrm{st}$ follow up and then further reduced to $9.1 \pm 10.0 \mathrm{ml}$ at second follow up. Thus volume reduction of thyroid gland was $42 \%$ at three months and $63 \%$ at six months.

Table 3: Comparison of thyroid volume in three types of Thyrotoxicosis

\begin{tabular}{|l|l|l|l|}
\hline Thyroid disease & \multicolumn{3}{|l|}{ Thyroid gland volume in $\mathrm{ml}$} \\
\hline & Base line & $\mathbf{1}^{\text {st }}$ follow-up & $2^{\text {nd }}$ follow-up \\
\hline GD (n=86) & $18.6 \pm 10.6$ & $10.3 \pm 6.5$ & $5.9=4.1$ \\
\hline Toxic MVG (n=21) & $41.5 \pm 41.3$ & $24.2 \pm 16.0$ & $17.7 \pm 14.4$ \\
\hline STG (n=10) & $25.0 \pm 18.0$ & $19.6 \pm 21.8$ & $13.5 \pm 16.6$ \\
\hline
\end{tabular}

GD-Diffuse Toxic Goiter or Grave's discasc, TMNG- Toxic multiple nodular goitcr, STG Single Toxic Nodular goiter

Table 4: Multiple Comparisons following ANOVA showing TGV reduction.

\begin{tabular}{|c|c|c|c|c|c|c|c|c|}
\hline & \multicolumn{4}{|c|}{$1^{\text {st follow up }}$} & \multicolumn{4}{|c|}{$2^{\text {nd }}$ follow up } \\
\hline Comparison & $\begin{array}{l}\text { Mean } \\
\text { Difference }\end{array}$ & Std. Erro & $95 \% \mathrm{CI}$ & p valuc & \begin{tabular}{|l} 
Mean \\
Difference
\end{tabular} & \begin{tabular}{c|c}
$\mathrm{c}$ & Std. Error
\end{tabular} & $95 \% \mathrm{CI}$ & peraluc \\
\hline GD vs. Toxic VING & 8.8 & 3.4 & $5,0-17,2$ & $0.034^{*}$ & 14.8 & 3.8 & $5,3,24,3$ & $.002^{*}$ \\
\hline GD vs. STG & 1.6 & 4.8 & $-9.7-13.0$ & 0.983 & 7.1 & 4.0 & $-3.7,17.7$ & 7.227 \\
\hline Toxic VNG vs. STG & -7.3 & 5.4 & $-20.3=5.8$ & 80.446 & -7.8 & 5.3 & $-20,8,5.3$ & .321 \\
\hline
\end{tabular}

GD Difituse Toxic Goiter or Grave's disease, MVG Multiple nodular goiter, STG Single Ioxic Nodular goiter The mean difference is significant at the 0.05 level.A VOVA: $\mathrm{F}=3.3 \mathrm{P}=.040$

While the mean TGV was found to decrease continually in each follow up in comparison to baseline, the mean FT4, though had an initial decline in 1st follow up, was seen to remain nearly unchanged during 2nd follow up, remaining in plateau. For the entire group of study patients the mean FT4 levels during baseline, 1st follow up and 2nd follow up were $35.3 \pm 16.6 \mathrm{pmol} / \mathrm{L}, 19.3 \pm 11.5$ $\mathrm{p} \mathrm{mol} / \mathrm{L}$ and to $20.3 \pm 8.1 \mathrm{pmol} / \mathrm{L}$. The laboratory normal range for FT4 assay was 9.5 to $25.5 \mathrm{p} \mathrm{mol} / \mathrm{L}$.

Since study patients were from three common clinical entities, GD, STG and toxic MNG, the comparison of $\mathrm{TGV}$ change among these three groups of patients were advantageously done. Table 3 shows the mean TGV among these three groups in each of three time points of TGV assessment by HRUS. Alike the cumulative trend the mean TGV was found to decrease continually in each follow up in comparison to baseline in each group of patients. 
ANOVA of volume reduction with post-hoq comparison through Hochberg test revealed statistically significant difference in thyroid volume reduction during 1st and 2nd follow-up between GD and toxic MNG while no statistically significant difference was observed among other pairs (Table 4).

\section{DISCUSSION}

The current study used an ellipsoid method for assessment of TGV using HRUS. This ellipsoid algorithm reported with the use of two orthogonal images, longitudinal and transverse scans, of each lobe $(18,19)$. The maximal length of a lobe in its three dimensions, namely cranio-caudal (c c), lateromedial (LM) and antero-posterior (AP) are measured followed by estimation of volume of that lobe using a cubic formula where: volume of lobe $\mathrm{V}=\mathrm{c} \mathrm{x} \mathrm{C} \mathrm{C} \mathrm{x}$ LM x AP was described (18). The constant ' $c$ ' in formula have reported values of 0.479 and 0.523 (or $\pi / 6)(20,21)$ with reports of higher accuracy using a value of 0.52 (22). Isthmus and pyramidal lobe (if present) were ignored in the current study following a report from Kuwait (23).

The current study restates the already proven role of RAIT to bring about a restoration of blood levels of FT4 and TSH hormones towards euthyroid picture with a concomitant alleviation of clinical manifestations of hyperthyroidism. The mean level of TSH was markedly increased during first followup and returned to near normal value during second follow-up due to replacement levothyroxine therapy. This was reflected by rise of baseline level of mean FT4 during 1st follow-up and then staying in a plateau during 2 nd follow-up.

The current study has found a decrement of TGV while performing serial measurements of TGV before RAIT followed by three and six months after RAIT in patients with hyperthyroidism. This finding is consistent with a previous report showing reduction of TGV up to $72 \%$ of initial volume within the first three months in 64 patients followed by continual size reduction till two years after RAIT (24). The objective and quantitative assessment of TGV by HRUS has gained acceptance as a standard routine procedure rather than assessment of thyroid enlargement by palpation during clinical visit (25). The current study attempted to explore further role of HRUS to determine reduction of $\mathrm{TGV}$ through serial measurement and comparison. Results of the current study indicate a correlative trend of TGV regression with normalization of FT4 and TSH blood levels. However, within the duration of study $23 \%$ patients retained hyperthyroid state and were scheduled for further RAIT. This indicates a need of further investigations to determine a cut-off value of TGV for response assessment with a potential implication in selection of patients for further RAIT. Thus, practice of HRUS measurement of TGV following RAIT is considered to have potential to be accepted as an adjunct to hormone assay for assessment of therapy response.

The current study comprised patients having GD, STG and toxic MNG with observation of regressive decrement of TGV in each sub-groups; being more decrement in GD than the other two entities. This finding is consistent with a report of pronounced TGV reduction in diffuse toxic goiter or GD patients by a mean of $76 \%$ after three years of treatment (26).

This phenomenon has been explained with the hypothesis that RAIT impart high dose of radiation to all thyroid cells in case of GD. In contrast toxic nodule of nodular goiter takes up most of the radioactive iodine causing its self destruction and concomitant protection of para-nodular normal tissue that receives small doses of radiation (27).

However, for selection of the small proportion of patient who shall remain unresponsive to single dose of RAIT and shall require further dose, determination of a cut off for TGV through further research, will be to be useful.

\section{CONCLUSION}

Single dose of RAIT was found to cause reduction of TGV in a larger proportion of patients. This rediscovers the capability of RAIT for non-invasive alleviation of clinical manifestations of hyperthyroidism avoiding the morbidity and poor cosmetic outcome of surgery as well as avoiding the risk of recurrence associated with ATD. In addition, the apparent correlative trend of TGV reduction with normalization of hormone levels indicates potentiality 
of HRUS to appear in an extended role of assessment of therapy response following RAIT beyond its conventional role in pre-therapy work-up.

\section{DISCLOSURE}

No competing financial interests exist

\section{REFERENCES}

1. Hussain R. History and Perspectives of Nuclear Medicine in Bangladesh. Asia Oceania J Nucl Med Biol 2016; 4(1): 55-8. doi: 107508/aojnmb.2016.04.009

2. Alam MN, Chakrabarty RK, Akhter M, Nahar N, Swapan MK, Alam MM, et al. Effectiveness of radioiodine therapy in treatment of hyperthyroidism. Mymensingh Med J 2013;22(4):632-9.

3. Mahmud MK, Jalil S, Rahman AM, Rashid MM, Sultana S, Taher A, et al. Etiologies and posttreatment conditions of thyrotoxic patients in Sylhet division, Bangladesh: A clinical series. Avicenna J Med 2017;7(3):125-9.

4. Paul AK, Miah SR, Mamun AA, Islam S. Thyroid disorders in Khulna district: a community based study. Bangladesh Med Res Counc Bull. 2006;32(3):66-71.

5. Lee SL, Ananthakrishnan S. Hyperthyroidism and Thyrotoxicosis. webMD LLC retrieved from www.emedicine.medscape.com/

6. Lazarus JH, Clarke S.Use of radioiodine in the management of hyperthyroidism in the UK: development of guidelines, Thyroid1997;7(2):229-31.

7. Franklyn JA, Maisonneuve P, Sheppard MC, Betteridge J, RNG, Boyle P. Mortality after the treatment of hyperthyroidism with radioactive iodine, $\mathrm{N}$ Engl $\mathrm{J}$ Med1998;338,(11):712-8.

8. Holm LE, Hall P, Wiklund K, Lundell G, Berg G, Bjelkengren $\mathrm{G}$ et al. Cancer risk after iodine-131 therapy for hyperthyroidism, J Natl Cancer Inst1991; 83(15):1072-7.

9. Peters H, Fischer C, Bogner U, Reiners C, Schleusener H.Radioiodine therapy of Graves' hyperthyroidism: standard vs. calculated 131I activity. Results from a prospective, randomized, multicentre study. Eur J Clin Invest 1995;25(3):186-93.

10. Wang J, Qin L.Radioiodine therapy versus antithyroid drugs in Graves' disease: a meta-analysis of randomized controlled trials. Br J Radiol. 2016:20160418. [Epub ahead of print]DOI: 10.1259/bjr.20160418

11. Stålberg P, Svensson A, Hessman O, Akerström G, Hellman P. Surgical treatment of Graves' disease: evidence-based approach. World J Surg. 2008;32(7):1269-77. doi: 10.1007/s00268-008-9497-9.

12. Ferrari C, Reschini E, Paracchi A.Treatment of the autonomous thyroid nodule: a review, Eur J Endocrinol 1996:135(4):383-90.doi:10.1530/eje.0.1350383

13. Hermus AR, Huysmans DA.Treatment of benign nodular thyroid disease. N Engl J Med.1998;338(20):1438-47.doi: 10.1056/NEJM199805143382007

14. Nygaard B, Faber J, Hegedüs L.Acute changes in thyroid volume and function following 131I therapy of multinodular goiter. Clin Endocrinol

(Oxf) 1994;41(6):715-8.

15. Bhattacharjee PK. 2000, The determination of thyroid volume by ultrasound and its relationship to body weight, age and sex in normal Bangladeshi subject., M Phil. Thesis, University of Dhaka, pp. 35.

16. Massol J, Pazart L, Aho S, Strauch G, Leclere J, Durieux P. Management of the thyroid nodule. Preliminary results of a practice survey of 685 general practitioners and specialists, Ann Endocrinol (Paris)1993;54(4):220-5.

17. Bruneton JN, Maestro CB, Marcy PY, Melia P, Mourou MY.Very high frequency (13 MHz) ultrasonographic examination of the normal neck: detection of normal lymph nodes and thyroid nodules. J Ultrasound Med 1994;13(2):87-90.

18. Brown MC, Spencer R.Thyroid gland volume estimated by use of ultrasound in addition to scintigraphy. Acta Radiol Oncol Radiat Phys Biol 1978;17(4):337-41.

19. Takalo RM, Mäkäräinen HP, KaarinaJaakkola R. Thyroid gland volume and echo structure in 13-year-old children in northern Finland. Acta Endocrinol (Copenh)1991;124(3):238-44.

20. Zimmermann P, Takala T, Poyhonen L, Punnonen R. Ultrasonography of the thyroid gland in pregnancies complicated by autoimmune thyroid disease. J Clin Ultrasound 1993;21(2):109-13.

21. Vitti P, Martino E, Aghini-Lombardi F, Rago T, Antonangeli L, Maccherini D et al. Thyroid volume measurement by ultrasound in children as a tool for the assessment of mild iodine deficiency. J Clin Endocrinol Metab 1994;79(2):600-3.doi: 10.1210/jcem.79.2.8045982

22. Reinartz P, Sabri O, Zimny M, Nowak B, Cremerius U, Setani $\mathrm{K}$ et al. Thyroid volume measurement in patients prior to radioiodine therapy: comparison between

three-dimentional magnetic resonance imaging and ultrasonography. Thyroid 2002;12(8):713-7. doi: $10.1089 / 105072502760258695$

23. Sheikh M, Doi SA, Sinan T, Al-Shoumer KA.Technical observations on the assessment of thyroid volume by palpation and ultrasonography. $\mathrm{J}$ Ultrasound Med 2004;23(2): 261-6.

24. Nygaard B, Hegedus L, Ulriksen P, Nielsen KG, Hansen JM. Radioiodine therapy for multinodular toxic goiter. Arch Intern Med1999; 159(12):1364-8.

25. Hegedus L, Perrild H, Poulsen LR, Andersen JR, Holm B, Schnohr P. et al. The determination of thyroid volume by ultrasound and its relationship to body weight, age, and sex in normal subjects. J Clin Endocrinol Metab 1983;56(2):260-3.doi: 10.1210/jcem-56-2-260

26. Tarantini B, Ciuoli C, Di Cairano G, Guarino E, Mazzucato P, Montanaro A et al.Effectiveness of radioiodine (131-I) as definitive therapy in patients with autoimmune and non-autoimmune hyperthyroidism. J Endocrinol Invest 2006;29(7):594-8.

27. Cooper DS.Radioiodine for hyperthyroidism: where do we stand after 50 years? JAMA 1998;280(4):375-6. 